\title{
Surface roughening and surface diffusion in kinetic thin-film deposition
}

\author{
Rong-Fu Xiao ${ }^{1}$ and Nai-Ben Ming ${ }^{2}$ \\ ${ }^{1}$ Department of Physics, The Hong Kong University of Science \& Technology, Clearwater Bay, Kowloon, Hong Kong \\ ${ }^{2}$ Center for Condensed Matter Physics and Radiation Physics, CCAST (World Laboratory), Beijing, China \\ and National Laboratory of Solid State Microstructures, Nanjing University, Nanjing 210008, People's Republic of China
}

(Received 15 December 1993)

\begin{abstract}
We have studied the correlation between surface roughening and surface diffusion in kinetic thin-film deposition by Monte Carlo simulation. Through a variation of simulation parameters, we have obtained an optimal deposition window for layer-by-layer growth in the parameter space of temperature and impingement rate. We have found that an atomically smooth surface can only be obtained at a regime of intermediate temperatures and low impingement rate, beyond which the growing surface is either kinetically rough (at low temperatures) or thermally rough (at high temperatures). The kinetic roughening can be avoided by choosing a lower deposition rate, but the thermal roughening cannot be compensated by any choice of particle impingement. We have shown that surface diffusion is closely correlated with surface roughening. Only when the surface diffusion length is long can a surface be grown in a layer-bylayer mode.
\end{abstract}

PACS number(s): 61.50.Cj, 68.55. - a, 68.35.Fx, 05.50.+q

Surface growth has recently received much interest in thin-film epitaxy [1-11]. The ability to grow an atomically smooth (sharp) interface is necessary in thin-film device applications. Depending on growth conditions, an initially flat crystal surface may grow normally when the surface is atomically rough or grow laterally (layer by layer) when the surface is atomically smooth [7]. The transition from lateral to normal growth (the so-called surface-roughening transition) can occur as substrate temperature and/or impingement rate are changed. In general, crystal surface is rough at either high temperatures (thermal roughening) or at low temperatures and/or for high deposition rates (kinetic roughening) [1-7]. Marmorkos and Das Sarma [4], who employed a ballistic deposition model, without considering particle evaporation and diffusion after being stuck on the surface, have shown that there are no unique dynamic exponents universally characterizing the low-temperature kinetically rough growth regime and that, in principle, one can achieve layer-by-layer growth at low temperatures by suitably lowering the deposition rate. Their results provide a qualitative guideline in the selection of experimental conditions in thin-film deposition.

Among various experimental parameters, surface diffusion is one of the most important factors in controlling surface-growth morphologies [4-13]. In general, a crystal surface is smoother when surface-diffusion length is longer and rough when it is absent [7]. The first comprehensive study of surface diffusion began with Burton, Cabrera, and Frank (BCF) [12]. Based on an equilibrium statistical model, $B C F$ showed that the surfacediffusion length $\lambda_{s}$ of an adsorbed (ad)atom is related to the crystal surface temperature as follows:

$$
\lambda_{s}=a \exp \left[\left(E_{i}-\delta E_{i}\right) / 2 k T\right],
$$

where $a$ is a lattice constant, $E_{i}$ is the evaporation energy of the adatom from surface to vapor, and $\delta E_{i}$ is the activation energy for surface diffusion. Since evaporation energy $E_{i}$ is always larger than the activation energy $\delta E_{i}$ for surface diffusion, BCF concluded that the surfacediffusion length should increase monotonically with decreasing temperature. Such a conclusion has been confirmed by some experiments [5] and by Monte Carlo (MC) simulations [7]. However, recent experiments using field-ion microscopy (FIM) [10] and reflection highenergy electron diffraction (RHEED) [11] have shown an opposite dependence of surface diffusion length on temperature. That is, the surface-diffusion length increases with rising temperature.

To discern the experimental discrepancy concerning the temperature dependence of surface-diffusion length and to study further the effect of surface diffusion on surface roughening, we have recently expanded our earlier effort of MC simulation [7] to a wider range of temperature and supersaturation. In this Brief Report we report some new results about the collective effect of temperature and impingement rate on surface roughening as well as surface diffusion. The MC model reported here is roughly the same as in our earlier work [7]. Here we discuss only the formulation differences. Basically, in the simulation, we have considered three microscopic events, namely, particle impingement, evaporation, and surface diffusion. The impingement is characterized by an impingement flux $J$, which takes the simple form

$$
J=J_{\text {eq }}(1+\alpha),
$$

where $J_{\text {eq }}$ is the equilibrium impingement flux and $\alpha$ is a parameter that characterizes the net deposition of particles with respect to $J_{\text {eq }}$. After impinging on the surface, particles can either remain at the original site, return to the vapor (evaporate), or continue to wander to an unoccupied neighboring site (surface diffusion). The rate of both evaporation and surface-diffusion processes are sen- 
sitive to the local configuration of the site from which a molecule is to be dislodged. Following our earlier work [7], we cast the evaporation rate into the site-dependent form

$$
K_{i}^{-}=v \exp \left(-E_{i} / k T\right),
$$

where $v$ is a lattice vibration factor and $E_{i}$ is the total interaction (evaporation) energy of the interfacial atom $i$ with its solid neighbors. To simplify the problem, in this work we have included only particle interactions with the nearest solid neighbors characterized by a bond strength $\phi$.

For surface diffusion, the hopping rate of an atom is simply determined by the Arrhenius kinetic equation

$$
K_{i \rightarrow j}=v_{s} \exp \left(-\delta E_{i} / k T\right),
$$

where $v_{s}$ is a surface vibration factor and $\delta E_{i}$ the activation energy $[4,6]$,

$$
\delta E_{i}=m \phi_{\perp}+\eta n \phi_{\|},
$$

with $\phi_{\perp}$ being the diffusion barrier associated with the underneath substrate $(m)$ atoms $(m=1$ for a simple cubic lattice and considering the first nearest-neighbor interaction only) and $\phi_{\|}$the bond strength with the lateral neighboring $(n)$ atoms, and $\eta(<1)$ a dimensionless parameter characterizing the effective contribution to the $\delta E_{i}$ from lateral bonds. This equation is based on the fact that a diffusing atom must completely break the vertical bond with the substrate atoms but only needs to partially break its lateral bonds (solid neighbors on the same layer). In our simulation, we have chosen $\eta=0.5$ and $\phi_{\perp}=\phi_{\|}=\phi$ (for homoepitaxy). The $v_{s}$, in general, is different from the $v$ in Eq. (3) for evaporation. However, in this simulation we have neglected the difference. The corresponding probabilities for attachment, detachment, and surface diffusion can be obtained from Eqs. (2)-(4) [7]. In this formulation, we have used the relation

$$
J_{\text {eq }} /\left(v / a^{2}\right)=\exp (-\phi / k T)
$$

instead of a kink site equilibrium assumption [7]. Thus, the final input parameters in the simulation are only $\alpha$ and $\phi / k T$ dependent. The improvement of our simulation as compared with the earlier work of Marmorkos and Das Sarma [4] is that we have included the event of evaporation throughout our simulation to take into account the frequent atom dislodges at high temperatures. Rather than a unit sticking probability [4], the sticking probability in our simulation is highly anisotropic. Also, no solid-on-solid (SOS) is assumed in our simulation, and overhangs and vacancies are all allowed.

The simulation is conducted in a square area of (at least) 100 lattice units wide with periodic boundary conditions in both the $x$ and the $y$ directions for singular faces, and a peculiar periodic condition with vertical shift along the $z$ direction for a vicinal surface. That is, if a molecule leaves from the right most low step, it will return to the left most top terrace at the same $y$ value. The actual shift is determined by the tilting angle of the vicinal surface. To mimic the high-vacuum condition and large mean free path of atoms in an UHV deposition environment, we have considered a direct random impingement of atoms from a planar source to the crystal surface. In the simulation, surface atoms are all considered to be movable until they are covered by late-coming atoms (i.e., become part of a bulk solid). The simulation continues until a preset number of atoms have been deposited.

The results in Fig. 1 show the effect of temperature on growth morphologies for a (001) surface of a simple cubic crystal at a fixed value of $\alpha=0.07$. As one can see, at very low temperature $\phi / k T=20$ [Fig. 1(a)], the surface is

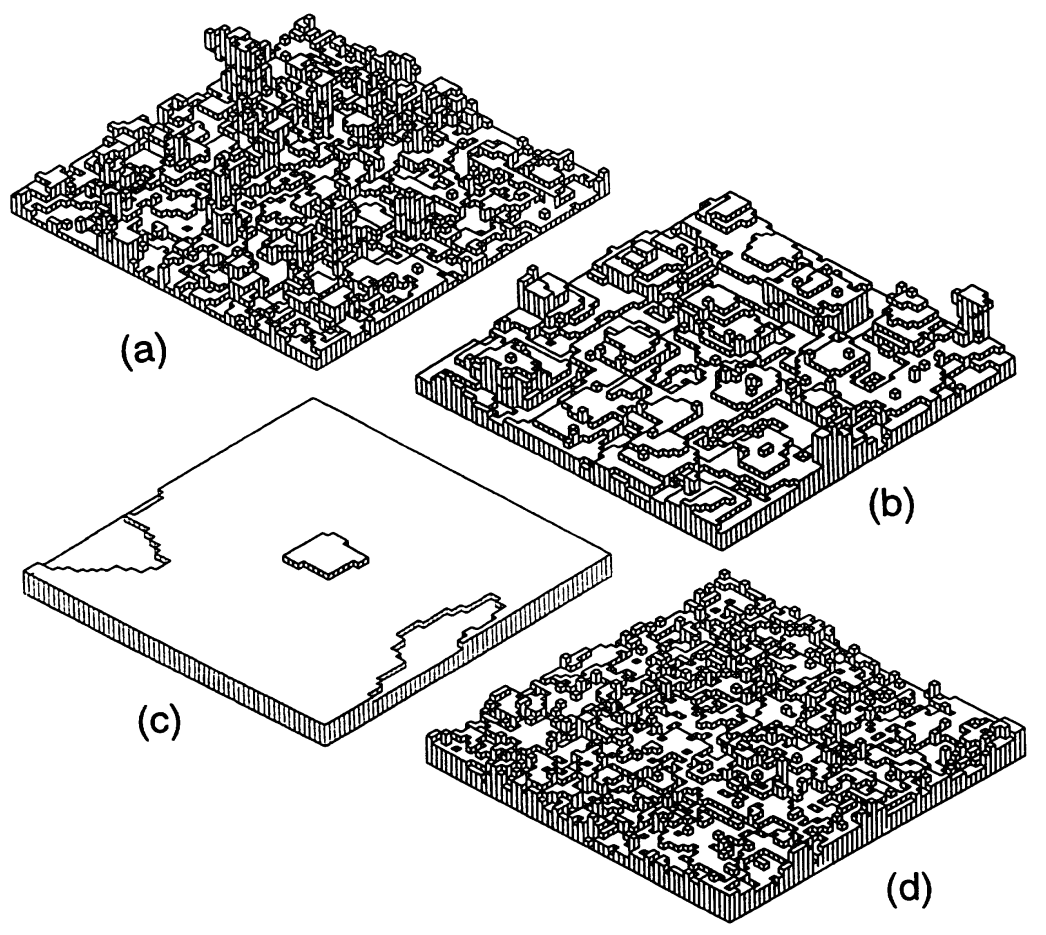

FIG. 1. Effect of temperature on surface growth morphologies at $\alpha=0.07$ : (a) $\phi / k T=20$, (b) $\phi / k T=14$, (c) $\phi / k T=8$, and (d) $\phi / k T=1.5$. 
extremely rough and its thickness expands to a wide extent (so-called kinetic roughening [2-4]). As temperature increases $\phi / k T=14$ [Fig. 1(b)], the crystal surface becomes smoother and flatter, and with further increase of temperature $\phi / k T=8$ [Fig. 1(c)], monolayer growth occurs (the best growth condition if a sharp interface is required). When the temperature reaches a critical value of $\phi / k T=1.5$ [Fig. 1(d)], thermal roughening appears [7]. Such a change of growth morphology has been seen in a $(1+1)$-dimensional model by Phillips and Chrzan [3]. By using a discrete-Gaussian model, these authors have found an optimal combination of growth rate and substrate temperature that minimizes the width of an interface ("interline" in their two-dimensional simulation).

By counting the number of dangling bonds from all interfacial layers, we have calculated surface roughness [12] at various temperatures and impingement rates. Using the same criterion as in our earlier MC simulation [7], we have obtained a transition boundary between smooth and rough surfaces in the parameter space of $\alpha$ and $\phi / k T$ (Fig. 2). As one can see from this figure, there exists an optimum growth window, and a crystal surface is atomically smooth only when it is grown at elevated temperatures and at relatively low impingement rates. Both high and low temperature will induce a rough surface. In comparison to the effect of temperature, an increase in particle impingement can always make a surface rougher. As one can see from Fig. 2, at a high-temperature regime a layer-by-layer growth mode cannot be obtained after growth temperature exceeds the thermal roughening transition point even at a very low deposition rate, due to a steep boundary there. However, at the lowtemperature regime, smooth layer growth is always possible by lowering the impingement rate further. The rule of thumb is that the lower the temperature, the slower the growth rate should be, in order to obtain lateral surface growth. The tradeoff between smooth surface and slow growth continues until the temperature reaches zero, in which case no smooth surface can be grown no matter how small the deposition rate is.

Surface roughness directly affects material transport on the surface. To estimate surface-diffusion length at different growth conditions, we have employed two different schemes. In the first scheme, we kept track of

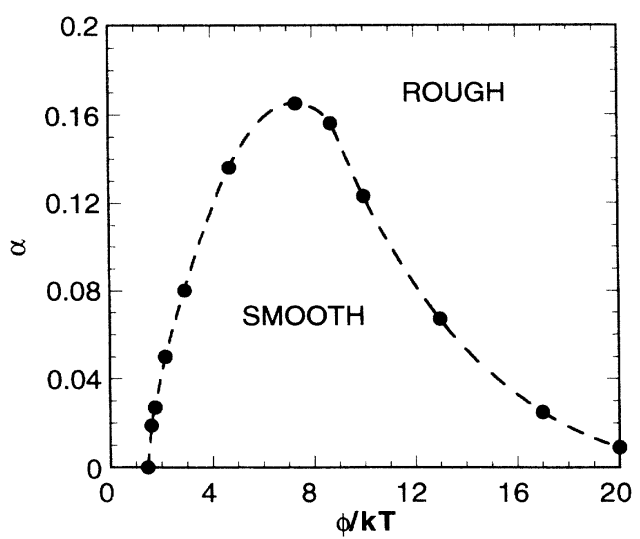

FIG. 2. Surface-roughening transition in the parameter space of $\alpha$ and $\phi / k T$. Surface is rough above the curve and smooth below. the actual pass of an individual atom as soon as it impinged onto the surface. The surface-diffusion length is calculated from the distances between the point where the atom lands on the surface and the point where the atom either sticks on the surface or evaporates back to the vapor phase. In the second scheme, we realized the fact that in vicinal surface growth two-dimensional nucleation (2DN) growth is unlikely if half of the terrace width is equal to or narrower than the surface-diffusion length [11-13]. Hence, at a given value of $\alpha$ and $\phi / k T$, we adjusted the width of the terrace [Figs. 3(a)-3(c)]. From the case when $2 \mathrm{DN}$ islands have just disappeared [Fig. 3(b)] we took half of the the terrace width as the surface-diffusion length for the given temperature and impingement flux. We found that the surface-diffusion length obtained from the first method is a little bit short-
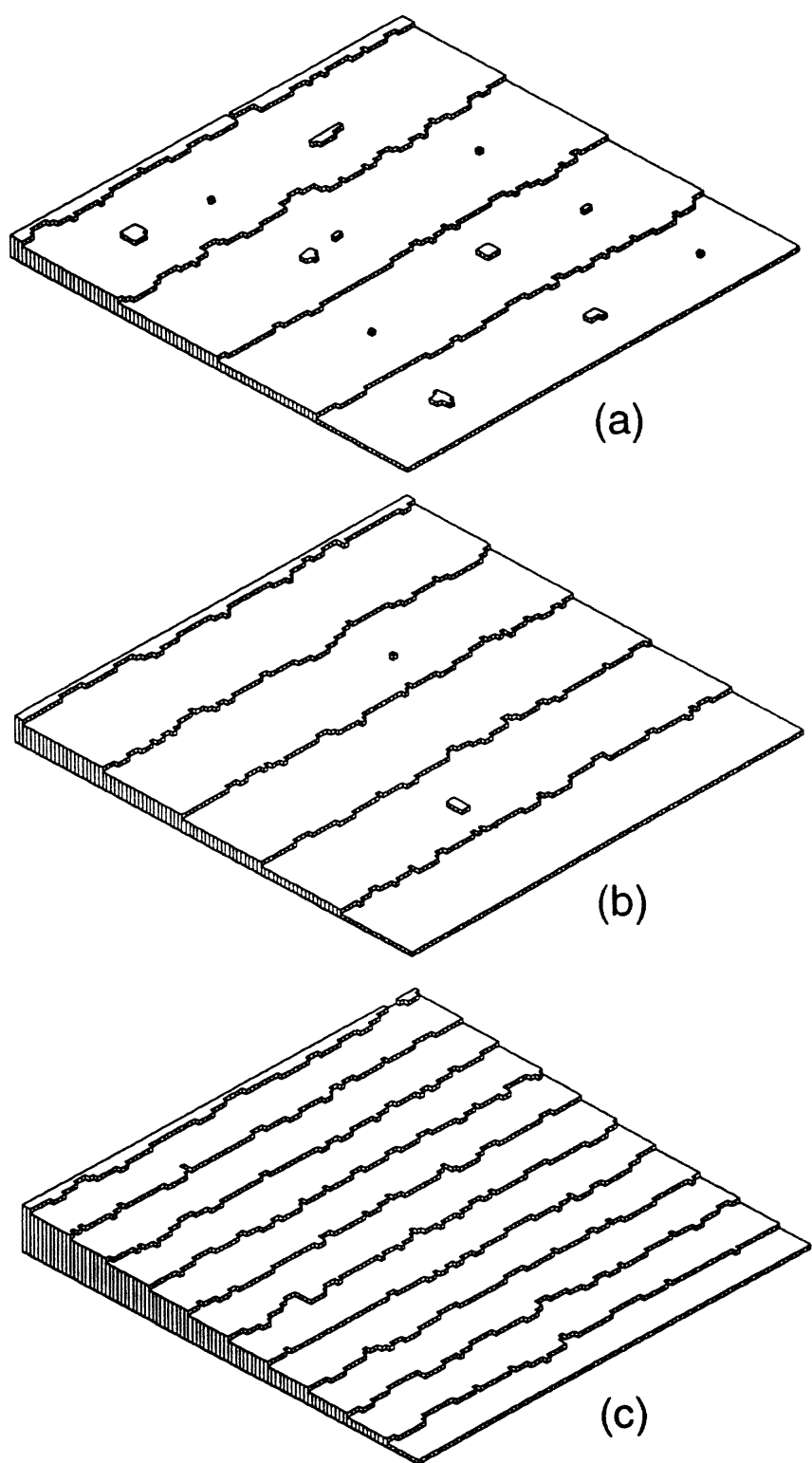

FIG. 3. Effect of terrace width $L$ (or orientation of vicinal surface) on 2DN and step flow (SF) [13] growth at $\alpha=0.1$ and $\phi / k T=5.5$ : (a) $L=25 a, 2 \mathrm{DN}$ 's are present; (b) $L=20 a$, 2DN's are greatly reduced and SF growth becomes dominant; (c) $L=10 a$, no $2 \mathrm{DN}$ 's, and the surface is grown by SF only. 
er $(\sim 0.8)$ than that from the terrace width calculation. The result in Fig. 4 shows surface-diffusion length dependence on temperatures at two different impingement rates: $\alpha=0.07$ (denoted by circles) and 0.1 (denoted by squares) that are obtained by using the second method. As one can see from this figure, the surface-diffusion length reaches a maximum value around $\phi / k T \sim 7.5$, and decreases to zero at both low and high temperatures. The decrease of surface-diffusion length at the two extreme temperature regimes stems from different growth mechanisms. At the high-temperature regime (left-hand side) the decrease of the $\lambda_{s}$ with increasing temperature is due to thermal roughening (thermally activated holes and islands prevent atoms from diffusing farther) and the decrease of the $\lambda_{s}$ at a low-temperature regime (right-hand side) results from kinetic roughening (atoms move very slowly and the relaxation is hindered by a low kinetic energy). At zero substrate temperature, no surface diffusion is possible even if there is only a very small deposition rate. In contrast to temperature effect, an increase in particle impingement (from $\alpha=0.07$ to 0.1 in Fig. 4) shortens surface-diffusion length at all temperature regimes. The result in Fig. 4 can be used to explain why surface-diffusion length increases in some experiments while it decreases in others. As we can see when the growth condition is near the kinetically controlled (lowtemperature) regime, the surface-diffusion length will increase with rising temperature $[10,11]$, and when the experiment is conducted at the thermally activated (hightemperature) regime, the surface-diffusion length will decrease with increasing temperature [5]. One should realize that there is a fundamental difference in surface diffusion between equilibrium and nonequilibrium situations. In an equilibrium situation atoms are supposed to be able to diffuse continuously until they find an energetically favorable position. However, during growth, atoms have only a finite diffusion time $\left(1 / J a^{2}\right)[8]$ beyond which they will be buried or blocked by late-coming particles. Such a difference in surface diffusion narrows at high temperatures and widens at low temperatures. Therefore,

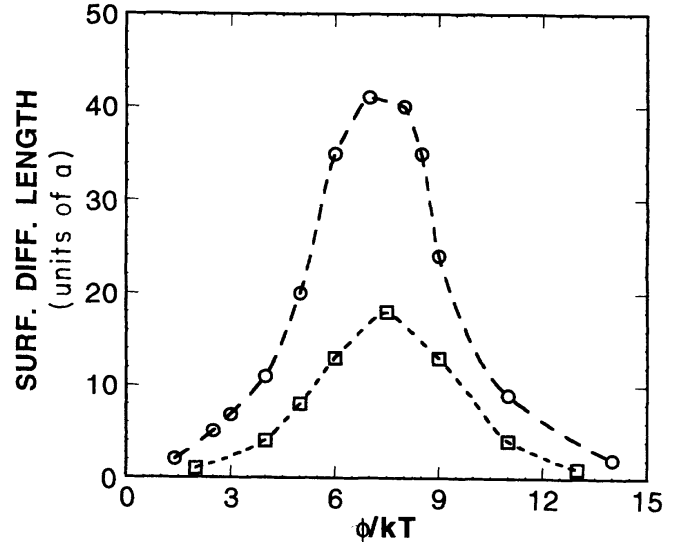

FIG. 4. Surface-diffusion length as a function of temperature (i.e., $\phi / k T)$ at $\alpha=0.07$ (circles) and 0.1 (squares).

BCF's surface-diffusion theory is applicable at quasiequilibrium situations or at high-temperature regimes if one wants to use it for nonequilibrium situations where the temperature (thermal) effect is dominant over the kinetic effect.

In conclusion, we have studied the correlation between surface roughening and surface diffusion in kinetic thinfilm growth by MC simulation. Through a variation of simulation parameters, we have obtained an optimal operation window for layer-by-layer growth in the parameter space of temperature and impingement rate. We have found that an atomically smooth surface can only be obtained at a regime with intermediate temperatures and low deposition rates, beyond which the growing surface is either kinetically rough (at low temperatures) or thermally rough (at high temperatures). Kinetic roughening can be avoided by choosing a lower deposition rate, but the thermal roughening cannot be overcome by any decrease in particle impingement. We have demonstrated that surface diffusion is closely correlated to surface roughening. Only when the surface-diffusion length is relatively long can a surface be grown in a layer-by-layer mode.
[1] See Kinetics of Ordering and Growth at Surface, edited by M. Lagally (Plenum, New York, 1990); in Evolution of Surface and Thin Film Microstructure, edited by H. A. Atwater, E. H. Chason, M. L. Grabow, and M. G. Lagally, MRS Symposia Proceedings No. 280 (Materials Research Society, Pittsburgh, 1992), and references therein.

[2] L.-H. Tang and T. Nattermann, Phys. Rev. Lett. 66, 2899 (1991); M. Uwaha and Y. Saito, ibid. 68, 224 (1992); M. A. Cotta, R. A. Hamm, T. W. Staley, S. N. G. Chu, L. R. Harriott, M. B. Panish, and H. Temkin, ibid. 70, 4106 (1993).

[3] R. Phillips, and D. C. Chrzan, Phys. Rev. Lett. 67, 220 (1991).

[4] I. K. Marmorkos and S. Das Sarma, Surf. Sci. 237, L411 (1990); S. V. Ghaisas and S. Das Sarma, Phys. Rev. B 46, 7308 (1992), and references therein.

[5] M. Tanaka, T. Suzuki, and T. Nishinaga, J. Cryst. Growth 111, 168 (1991); 111, 173 (1991); and references therein.

[6] C. N. Luse, A. Zangwill, D. D. Vvedensky, and M. R. Wilby, Surf. Sci. 274, L535 (1992); D. D. Vvedensky, A.
Zangwill, C. N. Luse, and M. R. Wilby, Phys. Rev. E 48, 852 (1993); D. D. Vvedensky, N. Haider, T. Shitara, and P. Smilauer, Philos. Trans. R. Soc. London, Ser. A 344, 493 (1993), and references therein.

[7] R.-F. Xiao, J. I. D. Alexander, and F. Rosenberger, Phys. Rev. A 43, 2977 (1991).

[8] Y. W. Mo, J. Kleiner, M. B. Webb, and M. G. Lagally, Phys. Rev. Lett. 66, 1998 (1991); E. Ganz, S. K. Theiss, I.-S. Hwang, and J. Golovchenko, ibid. 68, 1567 (1992).

[9] R. M. Tromp, A. W. Denler van der Gon, and M. C. Reuter (unpublished).

[10] M. F. Lovisa and G. Ehrlich, Surf. Sci. 246, 43 (1991); G. L. Kellogg, ibid. 246, 31 (1991), and references therein.

[11] J. H. Neave, P. J. Dobson, and B. A. Joyce, Appl. Phys. Lett. 47, 100 (1985).

[12] W. K. Burton, N. Cabrera, and F. C. Frank, Philos. Trans. R. Soc. London, Ser. A 243, 299 (1951).

[13] G. S. Bales, and A. Zangwill, Phys. Rev. B 41, 5500 (1990); R. Ghez and H. G. Cohen, Appl. Phys. Lett. 56, 1977 (1990). 